\title{
Microscopy assisted fabrication of a hydrogel-based microfluidic filter
}

A. Caliò

alessandro.calio@na.imm.cnr.it

\section{J. Leng}

\section{J. Decock}

\section{De Stefano}

\section{J. B. Salmon}

National Research Council - Institute for microelectronics and microsystems, Via P. Castellino 111, 80131, Naples, Italy

Dept. of Physical Science, University of Naples Federico II, Via Cinthia, 80100, Naples, Italy

Laboratory of the Future, avenue du Dr Schweitzer 178, F-33608, Pessac, France

Laboratory of the Future, avenue du Dr Schweitzer 178, F-33608, Pessac, France

National Research Council - Institute for microelectronics and microsystems, Via P. Castellino 111, 80131, Naples, Italy

Laboratory of the Future, avenue du Dr Schweitzer 178, F-33608, Pessac, France

A porous filter is fabricated directly inside a microfluidic circuit using a photoreticulable hydrogel. The filter could be used for separation of cells from blood, removal of particles or solutes, such as proteins, in microdialysis and microfiltering. The filter is realized by in situ polymerization approach: a liquid hydrogel is injected in a microfluidic circuit channel where the filter is formed in a specific location by polymerization of UV light, focused by an optical microscope.

[DOI: http://dx.doi.org/10.2971/jeos.2015.15058]

Keywords: Filtration, hydrogel, microfluidics

\section{INTRODUCTION}

Since 1990, microfluidics has nowadays developed into a versatile technology with a huge plethora of applications. While initially focused on liquid and gases flow through simple channel layouts, designs of chips are much more complicated since they could include lot of components integrated inside. Large effort has been spent into the integration of unit operations on-chip, e.g. sample pre-treatment, mixing of different substances, controlled chemical reaction, and separation/purification of products [1, 2]. Looking at methods used for components integration, many research groups have started out with clever designs of silicon chips, using the well-established toolbox of semiconductor industry. Lately, a shift toward new approaches increased, aimed to simple straightforward integration: mainly application of functionalized coatings, adsorption beads and filters. The use of filters in microfluidics is a topic of growing interest. Filter science and technology is a broad and highly interdisciplinary field, where process engineering, material science and chemistry meet all together. Exploring the boundaries of these fields offers many opportunities, and filters have already been used for an impressive range of functions, such as separation, purification, dialysis, and so on $[3,4]$. Traditional methods for improving components that can perform separation inside a microchannel include fabricating a barrier with reduced channel width [5] or an array of posts [6]. While these approaches are capable of providing a reusable filter, the fabrication process can be time consuming and even expensive. Other way to fabricate a filter is to create a packed bed column, either by packing beads [7] or by in situ polymerization [8]. The advantage of in situ polymerization is that the filter can be fabricated in a specific location without the need of a constriction region or other mechanisms that are commonly used to hold the beads. An additional benefit of this method is its application in existing chip formats (provided that the used chip material is transparent to UV light). Filters can be fabricated using many materials from both the hard and soft realms, such as silica, silicon, hydrogels and so on [9]-[12]. Hydrogels are particularly attractive because of their biocompatibility and due to the possibility of photo-induced cross-linking by simply adding a photoinitiator in the polymer solution, and thus they offer a new way to develop original fabrication protocols.

In this work, we report preliminary results on the fabrication and the characterization of a filter made by photoreticulable hydrogel within a microfluidic circuit.

\section{RESULTS AND DISCUSSION}

Most of the microfluidic devices are made of polydimethysiloxane (PDMS), since it combines numerous excellent physical and chemical properties [13, 14]. Nevertheless, some of the properties of PDMS strongly limit its range of applications. For example, this silicon elastomer swells in most organic solvents that is the major constraint to its use in aqueous solutions. Moreover, permanent modification of 
(A)

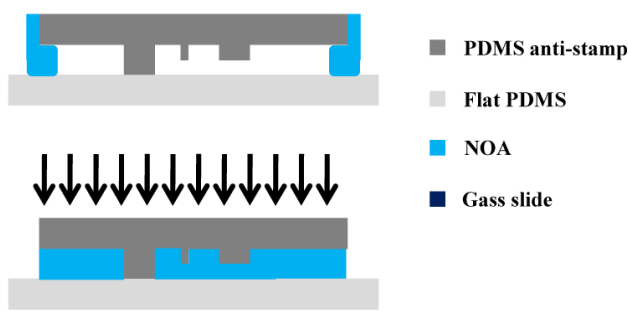

(C)

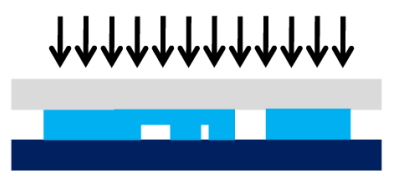

(D)

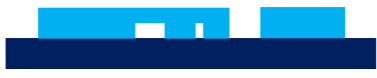

FIG. 1 (A) PDMS anti-stamp and flat PDMS are put in contact and the gap space between them is filled by NOA liquid. (B) The gap is spontaneously filled due to capillary forces and exposed to UV light. (C) The flat PDMS with the pattern above is put in contact with a glass slide and exposed to UV light. (D) Removal of the flat PDMS.

PDMS surfaces chemical properties is still a challenging task. Finally, the low elastic modulus of PDMS does not allow the use of high pressure $(>1 \mathrm{Bar})$ operations. In order to overcome these problems, we fabricated microfluidic circuits by using Norland Optical Adhesive (NOA). This is a single component liquid adhesive that can be cured in a few second of exposure to ultraviolet light. The fabrication process of microfluidic devices made in NOA has been described in details elsewhere (see in particular ref. [15]). In Figure 1, we briefly resume main production steps:

The starting object is the PDMS anti-stamp, that is the opposite of the PDMS stamp fabricated by soft lithography replica molding technique [16]. The anti-stamp is put in contact with a flat PDMS and then the empty space between them is filled with the liquid NOA (Figure 1(a)). In practice, the gap is spontaneously filled due to capillary forces (Figure 1(b)). To speed up the capillary spreading, the parts of the anti-stamp surrounding the pattern can be decorated with a dense network of pillars. This porous structure enhances the local curvature of the liquid meniscus and consequently the Laplace pressure, which drives the liquid motion, becomes higher. After exposure to UV light for 10 seconds at $40 \mathrm{~mW} / \mathrm{cm}^{2}$ (Figure 1(b)), the anti-stamp is removed. Since oxygen inhibits the freeradical polymerization used here to build the polymer network, the permeability to gas of the PDMS anti-stamp ensures that an ultra thin superficial layer of liquid remains uncured. In order to fix this phenomenon, the flat PDMS with the pattern above is put in contact with a glass slide and exposed to UV light for 10 minutes at $40 \mathrm{~mW} / \mathrm{cm}^{2}$ (Figure 1(c)). The exposure time is so high to strongly harden the NOA structure. The flat PDMS is then removed and the NOA microfluidic circuit is obtained above the glass slide (Figure 1(d)). Nanoports, which are plastic connectors that allow sealing plastic tubes for the injection of the liquids, are glued on the inlets and outlets of the circuit.

The material used to fabricate the filter is an aqueous hydrogel solution; more precisely, it is a mixture of $50 \% \mathrm{v} / \mathrm{v}$ poly(ethylene glycol) diacrylate 575 , photoinitiator $5 \% \mathrm{v} / \mathrm{v}$

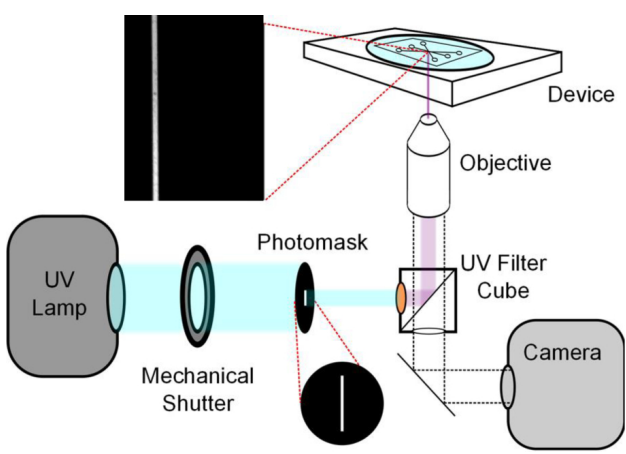

FIG. 2 Simplified microscope schematic for filter fabrication.

2-hydroxy-2-methylpropiophenone and $45 \%$ v /v water. Since this solution is completely transparent and it can be hardly seen in the microchannels, $2 \mu \mathrm{L}$ of carboxylate yellow-green fluospheres ( $1 \mu \mathrm{m}$ diameter on average) have been added for imaging purposes. In this way the filter, made by exposing this fluorescent solution to UV light, would be observable in fluorescence imaging, and thus it could be characterized by a microscope used in fluorescence mode. The circuit, constituted by two channels, is filled by the hydrogel solution; then, only a specific area of the circuit is exposed and polymerized by UV light. For the exposure, we used a microscope modified as it is reported in ref. [17]. Figure 2 shows a sketch of the setup used for the fabrication of the filter:

The microfluidic device is placed on the microscope stage and a photomask is inserted into microscope field aperture (see scheme in Figure 2). Natural fluorescence of NOA to the UV light allows the alignment between the patterned UV light and the area to be exposed. The solution is irradiated for $1 \mathrm{sec}-$ ond at $47 \mathrm{~mW} / \mathrm{cm}^{2}$. Hydrogel solution that was not polymerized, was then removed by flushing channels with water for several minutes. The fabrication of the filter can be also done by using a typical mask-aligner for standard photolithography or a microscope coupled to a Digital Micromirror Device (DMD) that does not require the photomask.

The final circuit is thus constituted by two channels (height $=25 \mu \mathrm{m}$, width $=225 \mu \mathrm{m}$ ) separated by the filter with height $=25 \mu \mathrm{m}$ and width $=50 \mu \mathrm{m}$ (Figure 3(a) $-(\mathrm{c})$ ).

A preliminary test has been done to characterize the working principle of the filter (see Figure 4 and Figure 5) by fluorescence microscopy. Figure 4 shows some kinetic properties of the filter, reported as contrasted images in Figure 4(a), and as fluorescence intensity graphic in Figure 4(b). The two channels are filled with water at $500 \mu \mathrm{L} / \mathrm{min}$ for some minutes in order to clean them. Then, inlet and outlet of the top channel are closed in order to stop the flow and the channel stays filled by water. Since they contain only water, the two channels are not fluorescent (Figure 4(a) I).The bottom channel has thus been filled by an aqueous solution of Rhodamine 6G (R6G) at $500 \mu \mathrm{L} / \mathrm{min}$, costant for all the experiment duration, and therefore the bottom channel became fluorescent (Figure 4(a) II). As time passed, the fluorescence of the bottom channel slowly oscillated, due to diffusion dynamic of R6G molecules when they passed through filter into the water of the top channel: the filter has a nanostructured morphology 
(A)

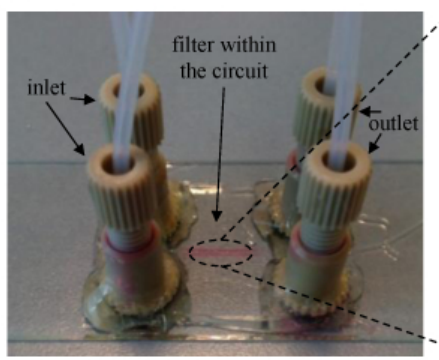

(B)

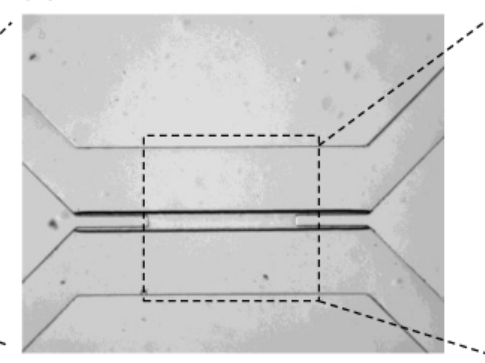

(C)

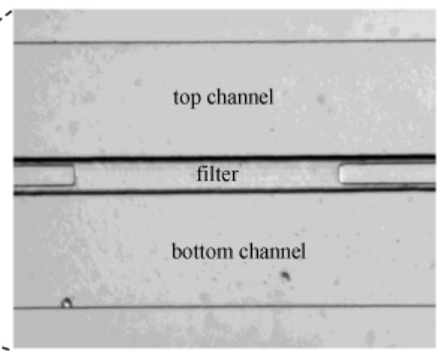

FIG. 3 (A) Sketch of the microfluidic device. (B) Zoom of the circuit. (C) Zoom of the filter: the filter separates the top channel from the bottom channel.

(A)

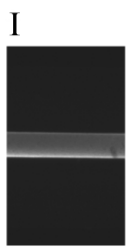

0

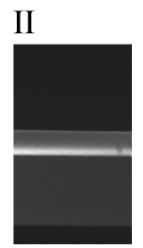

$65 \mathrm{~s}$

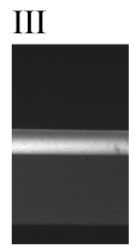

$135 \mathrm{~s}$

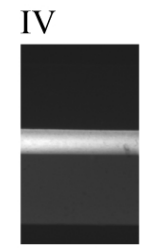

$220 \mathrm{~s}$

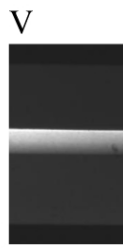

$570 \mathrm{~s}$
(B)

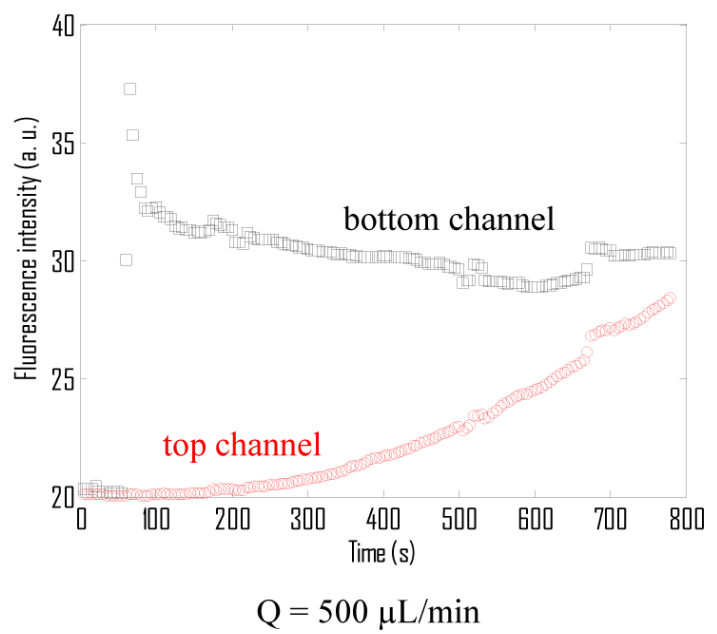

FIG. 4 Kinetics of the filter at $500 \mu \mathrm{L} / \mathrm{min}$ : fluorescence images (A) and intensities (B) of the two channels as function of time.

that makes non-linear the diffusion of the marker between the two channels. The R6G solution diffused from the bottom to the top channel through the filter (Figure 4(a) III, IV), therefore the top channel also became fluorescent (Figure 4(a) V), and a stationary condition, characterized by almost the same value of fluorescence intensity in both channels, was reached in less than 14 minutes.

Figure 4(b) quantifies the fluorescence intensities of the bottom and top channels during the experiment as function of the time. The intensities of the two channels tend to become equals after about 14 minutes.

Figure 5(a) and Figure 5(b) show the reversibility of the filter: at the beginning of the measurement, the two channels are fluorescents, due to the amount of R6G solution from the previous experiment (Figure 5(a) I). When the bottom channel is filled with water at $500 \mu \mathrm{L} / \mathrm{min}$, it rapidly becomes not fluorescent (Figure 5(a) II). As time passes, the R6G solution diffuses from the top to the bottom channel through the filter (Figure 5(a) III), and it is washed away; therefore, the fluo-
(A)

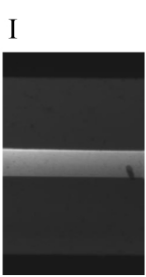

0

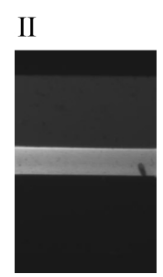

$85 \mathrm{~s}$

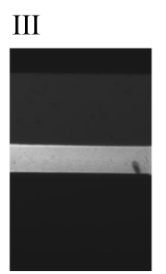

$715 \mathrm{~s}$

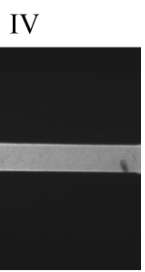

$1995 \mathrm{~s}$
(B)

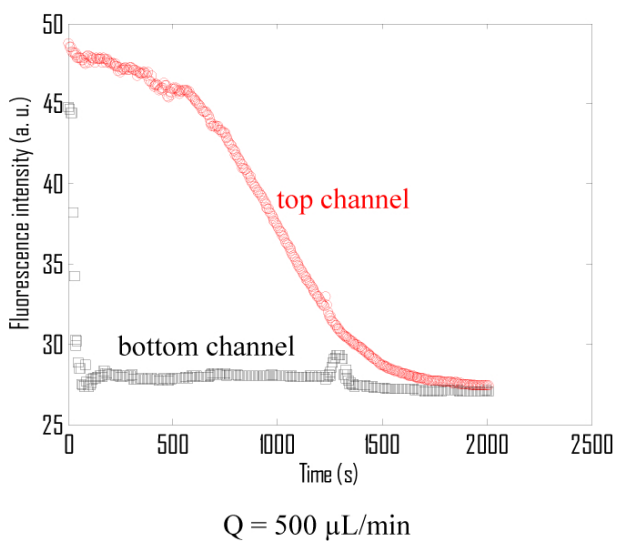

FIG. 5 Reversibility of the filter at $500 \mu \mathrm{L} / \mathrm{min}$ : fluorescence images (A) and intensities (B) of the two channels as function of time.

rescence intensity of the top channel decreases until becomes equal to the one of the bottom channel (Figure 5(a) IV).

Figure 5(b) shows the fluorescence intensities as function of the time of the bottom and top channels during the experiment. The intensities of the two channels tend to become equals in less than 34 minutes.

The same study has been performed by turning the solution flux at $100 \mu \mathrm{L} / \mathrm{min}$ (Figure 6 and Figure 7): the behavior of the system is very similar to the previous one and significant differences cannot been seen.

\section{CONCLUSIONS}

Preliminary results on fabrication and characterization of a hydrogel filter-based microfluidic circuit have been reported. A simple and fast fabrication method, based on in situ polymerization, has been used to make a hydrogel based filter inside a NOA microfluidic circuit.The main advantages of this method are its versatility, due to the possibility of fabricating 
(A) ${ }_{\mathrm{I}}$
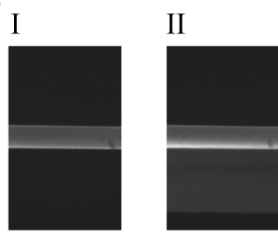

0

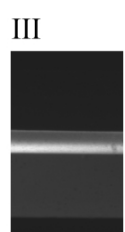

$95 \mathrm{~s}$

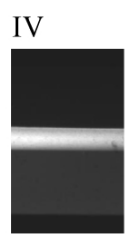

$235 \mathrm{~s}$

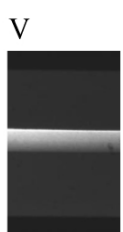

$650 \mathrm{~s}$

(B)

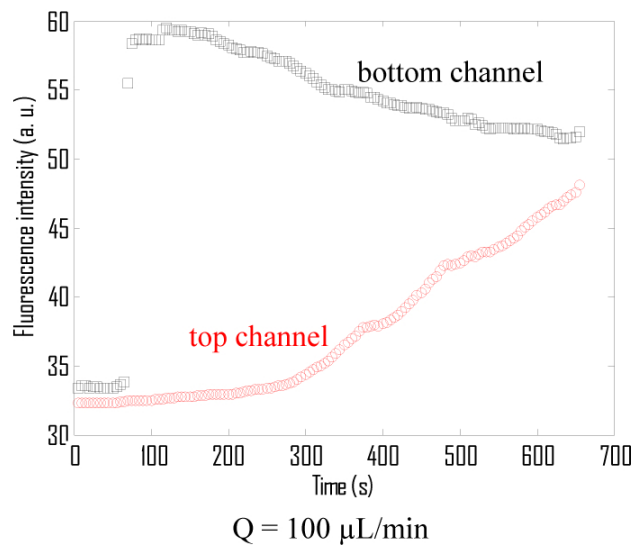

FIG. 6 Kinetics of the filter at $100 \mu \mathrm{L} / \mathrm{min}$ : fluorescence images (A) and intensities (B) of the two channels as function of time.

(A)

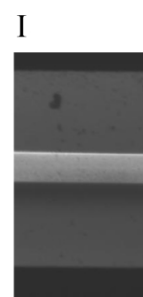

0

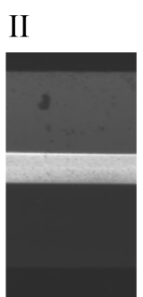

$570 \mathrm{~s}$

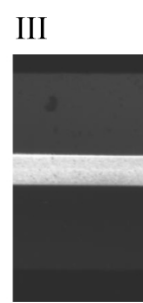

$1440 \mathrm{~s}$

IV

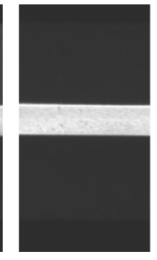

$3180 \mathrm{~s}$

(B)

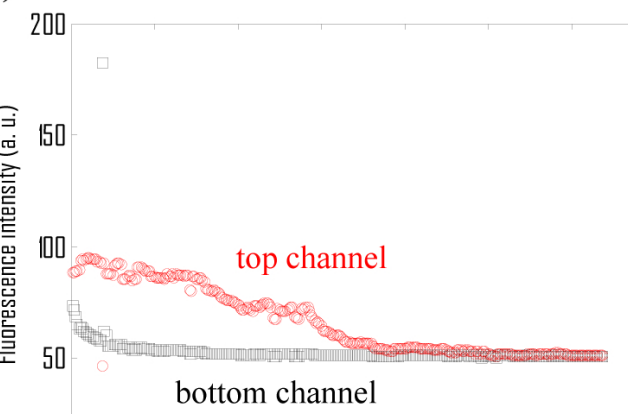

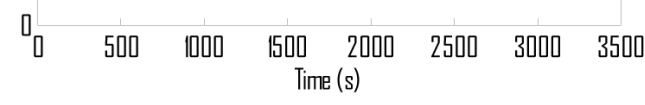

$$
\begin{aligned}
& \mathrm{Q}=100 \mu \mathrm{L} / \mathrm{min}
\end{aligned}
$$

FIG. 7 Reversibility of the filter at $100 \mu \mathrm{L} / \mathrm{min}$ : fluorescence images (A) and intensities (B) of the two channels as function of time.

the filter in any location of any microfluidic circuit, and the possibility to use several standard UV exposure systems, like a microscope coupled with a UV lamp, a mask aligner traditionally used for photolithography or a microscope coupled to a Digital Micromirror Device (DMD) and a UV lamp. Moreover, we verified that fluorescent molecules are able to diffuse through the filter and that its kinetics does not depend on the flow rate. The next step will consist in providing quantitative parameters of the filter, for example its permeability, by measuring the flow rate and the diffusion time.

\section{References}

[1] D. Erickson, and D. Li, "Integrated microfluidic devices," Anal. Chim. Acta 507, 11-26 (2004).

[2] K. Sato, A. Hibara, M. Tokeshi, H. Hisamoto, and T. Kitamori, "Microchip-based chemical and biochemical analysis systems," Adv. Drug Deliver. Rev. 55, 379-391 (2003).

[3] P. N. Floriano, N. Christodoulides, D. Romanovicz, B. Bernard, G. W. Simmons, M. Cavell, and J. T. McDevitt, "Membrane-based on-line optical analysis system for rapid detection of bacteria and spores," Biosens. Bioelectron. 20, 2079-2088 (2005).

[4] H. Mohamed, A. P. Russo, D. H. Szarowski, E. McDonnell, L. A. Lepak, M. G. Spencer, D. L. Martin, et al., "Development and characterization of on-chip biopolymer membranes," J. Chromatogr. A 1111, 214-219 (2006).

[5] J. P. Brody, T. D. Osborn, F. K. Forster, and P. Yager, "A planar microfabricated fluid filter," Sensor Actuator. A-Phys. 54, 704-708 (1996).

[6] B. He, L. Tan, and F. Regnier, "Microfabricated filters for microfluidic analytical systems," Anal. Chem. 71, 1464-1468 (1999).

[7] G. L. Lettieri, A. Dodge, G. Boer, N. F. de Rooij, and E. Verpoorte, "A novel microfluidic concept for bioanalysis using freely moving beads trapped in recirculating flows," Lab Chip 3, 34-39 (2003).

[8] F. Svec, E. C. Peters, D. Sykora, C. Yu, and J. M. J. Frechet, "Monolithic stationary phases for capillary electrochromatography based on synthetic polymers: designs and applications," J. High Res. Chromatogr. 23, 3-18 (2000).

[9] R. W. Tjerkstra, J. G. E. Gardeniers, J. J. Kelly, and A. Van den Berg, "Multi-Walled Microchannels: Free-Standing Porous Silicon Membranes for Use in $\mu$ TAS," J. Microelectromech. S. 9, 495-501 (2000).

[10] J. Khandurina, S. C. Jacobson, L. C. Waters, R. S. Foote, and J. M. Ramsey, "Microfabricated Porous Membrane Structure for Sample Concentration and Electrophoretic Analysis," Anal. Chem. 71, 1815-1819 (1999).

[11] S.-Y. Cheng, S. Heilman, M. Wasserman, S. Archer, M. L. Shuler, and M. Wu, "A hydrogel-based microfluidic device for the studies of directed cell migration," Roy. Soc. Ch. 7, 763-769 (2007).

[12] S. Klatt, M. Allerdißen, R. Körbitz, B. Voit, K.-F. Arndt, and A. Richter, "Hydrogel-based microfluidic systems," Adv. Sci. Tech. 81, 90-95 (2013).

[13] D. Psaltis, S. R. Quake, and C. Yang, "Developing optofluidic technology through the fusion of microfluidics and optics," Nature 442, 381-386 (2006).

[14] G. C. Randall, and P. S. Doyle, "Permeation-driven flow in poly(dimethylsiloxane) microfluidic devices," P. Natl. Acad. Sci. USA 102, 10813-10818 (2005).

[15] D. Bartolo, G. Degré , P. Nghe, and V. Studer, "Microfluidic Stickers," Lab Chip 8, 274-279 (2008).

[16] J. C. McDonald, D. C. Duffy, J. R. Anderson, D. T. Chiu, H. Wu, 0. J. A. Schueller, and G. M. Whitesides, "Fabrication of microfluidic systems in poly (dimethylsiloxane)," Electrophoresis 21, 27-40 (2000).

[17] J. S. Paustian, R. N. Azevedo, S.-T. B. Lundin, M. J. Cilkey, and T. M. Squires, “Microfluidic Microdialysis: Spatiotemporal Control over Solution Microenvironments Using Integrated Hydrogel Membrane Microwindows," Phys. Rev. X 3, 041010 (2013). 